\title{
Characterizing the Calcination Behaviors of Ni-Fe Layered Double Hydroxide Materials via In-situ Transmission Electron Microscopy
}

\author{
Christopher Hobbs ${ }^{1,3}$, Sonia Jaskaniec ${ }^{2,3}$ and Valeria Nicolosi ${ }^{2,3}$ \\ 1. School of Physics, Trinity College Dublin, Dublin, Ireland. \\ 2. School of Chemistry, Trinity College Dublin, Dublin, Ireland. \\ 3. Advanced Materials and Bioengineering Research (AMBER), Trinity College Dublin, Dublin, Ireland.
}

Layered double hydroxides (LDHs), a member of the two-dimensional nanomaterial category, are considered as versatile materials due to broad applications in medicine [1], catalysis [2] and flame retardants [3]. Moreover, LDHs have been extensively utilized as precursor materials for catalytic applications where the calcined layered materials results in the generation of catalytically active materials with applications in photocatalysis [4], hydrogen production [5] and magnetic materials [6]. However, a detailed description and observation of the nanoscale thermal evolution mechanisms has yet to be revealed. Herein, we report an in-situ transmission electron microscopy (TEM) characterization of Ni-Fe LDHs, revealing the nanoscale mechanisms involved with the thermal evolution of these versatile nanomaterials [7].

The LDH materials were studied by (scanning) transmission electron microscopy using a 300kV FEI Titan. In-situ heating experiments were conducted using an in-situ heating TEM sample holder (DENS solutions, The Netherlands). The precursor LDH platelets were first characterized by TEM and selected area electron diffraction (SAED) before subject to heated environments via an incremental heating ramp of $250^{\circ} \mathrm{C}$ for 2 hour, $450^{\circ} \mathrm{C}$ for 2 hours and finally $850^{\circ} \mathrm{C}$ for 2 hours. Subsequently, the properties of the calcined samples were post-analyzed in the TEM via energy filtered TEM (EFTEM), as well as energy dispersive x-ray spectroscopy (EDX) and electron energy loss spectroscopy (EELS) using scanning TEM (STEM).

Figure 1 presents bright field TEM images and corresponding selected area electron diffraction (SAED) patterns from various stages of in-situ heating experiments. Initially the Ni-Fe LDH material was found to have a hexagonal morphology with a well-defined crystal structure of hexagonal symmetry, typical of an LDH material (Figure 1(a)). As the platelets were subject to the incremental heating ramp, both a morphological and crystallographic transformation occurred. At $450^{\circ} \mathrm{C}$, the $\mathrm{LDH}$ material appeared to have an altered morphology, with a nucleation of spherical nanoparticles on the platelet surface (Figure 1(b)). Moreover, SAED analysis at this temperature indicated a loss of certain reciprocal lattice vectors, indicating a transition towards an amorphous phase. At $850^{\circ} \mathrm{C}$, the $\mathrm{LDH}$ materials thermally evolved into a confined array of spherical nanoparticles as a result of further nucleation, along with a change in crystallographic structure (Figure 1 (c) and (f)). Post-analysis of the calcined material showed that these spherical particles were predominantly composed of Nickel, as shown by EDX mapping in STEM (Figure 2 (c)). Also, these spherical particles were surrounded by Iron containing materials, highlighted by EELS mapping of the Iron $\mathrm{L}_{3}$ edge (Figure 2(d)).

The application of in-situ transmission electron microscopy revealed the nanoscale mechanisms involved in the thermal evolution of Ni-Fe LDH nanomaterials. The LDH precursor platelets were found to evolve into an array of coexisting Ni-containing particles amongst an Iron-containing matrix, confirmed by EFTEM as well as STEM-EDX and STEM-EELS mapping techniques [8]. 


\section{References:}

[1] Rives V, Arco M and Martín C, J. Control. Release, 169 (2013), p. 28.

[2] Xu Z P et. al, Appl. Clay Sci. 53 (2011), p. 139.

[3] Gao Y et. al, J. Mater. 2 (2014), p. 10996.

[4] Yuan X et. al, Appl. Clay Sci. 143 (2017), p. 168.

[5] Abelló S, Bolshak E and Montané D, Appl. Catal. A Gen. 450 (2013), p. 261.

[6] Zhou T et.al, Acta Chim. Sin. 60 (2002), p. 1078.

[7] Hobbs C et. al, npj 2D Mater. Appl, accepted article 15 ${ }^{\text {th }}$ January 2018.

[8] The authors would like to thank the Advanced Microscopy Laboratory, Trinity College Dublin, Ireland for provision of their facilities. The funding agencies, SFI AMBER, SFI PIYRA, ERC StG 2D NanoCaps, ERC CoG 3D2DPrint and Horizon2020 NMP Co-Pilot are also thanked for their support.

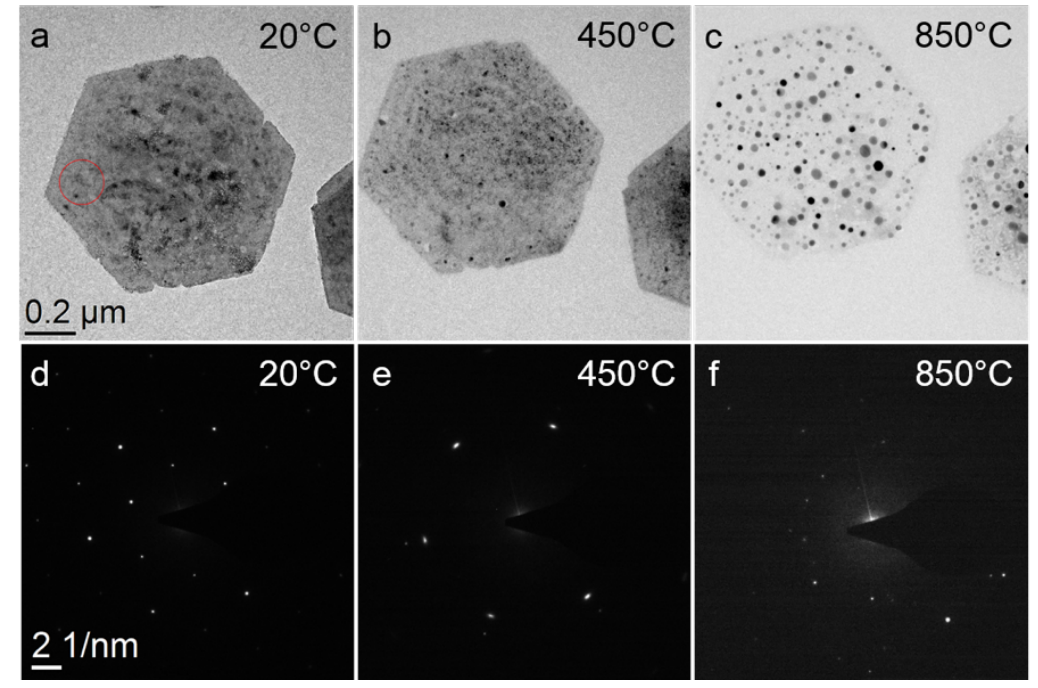

Figure 1. (a)-(c) Bright field TEM images and corresponding (d)-(f) selected area electron diffraction patterns from various steps of the in-situ heating of the Ni-Fe LDH nanomaterial. Red circle in (a) indicates acquisition region of associated electron diffraction patterns.

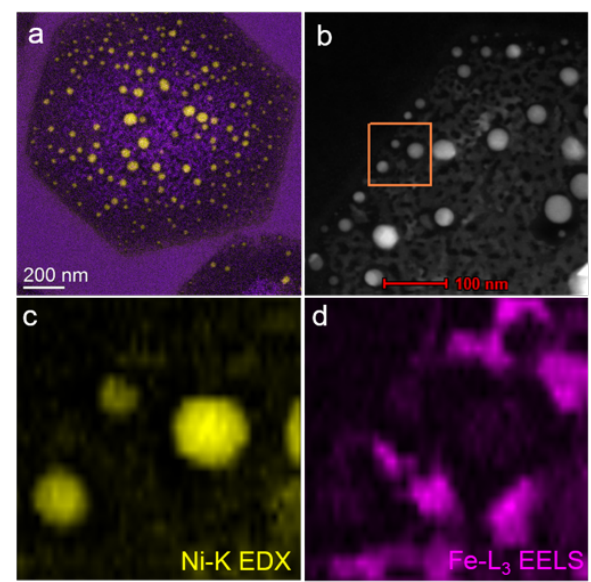

Figure 2. (a) Composite Ni (yellow) and Fe (purple) EFTEM maps of the calcined LDH platelet. (b) STEM image of calcined LDH material. Orange square indicates STEM-EDX/STEM-EELS map acquisition area. (c) Ni-K EDX map. (d) Fe-Ls EELS map. 